\section{Besondere Form einer Rachitis mit ungewöhnlichem, Malignität vortäuschen- dem braunen Tumor}

Braune Tumoren bei einem sekundären Hyperparathyreoidismus entstehen am wachsenden Skelett im Wesentlichen dort, wo es zu starken mechanischen Belastungen kommt - sie sind ebenso wie fibröse metaphysäre Defekte im Insertionsbereich von Muskeln, vorwiegend an der unteren Extremität lokalisiert. Durch Akzeleration des lokalen Knochenabbaus entstehen Höhlen, in die es einbluten kann. In der Folge bilden sich reaktive paraossale Veränderungen aus. Diese Epiphänomene können insbesondere in der MR-Bildgebung einen malignen Knochentumor vortäuschen.

\section{Fallbeschreibung}

Ein 11 Jahre altes Mädchen arabischer Herkunft in traditioneller Kleidung wurde mit Schmerzen und einer Schwellung am distalen linken Oberschenkel unter der Vorstellung eines aggressiven Knochentumors konsiliarisch in unsere Klinik eingewiesen.

Die konventionellen Aufnahmen des linken Knies (Abb.1) zeigen eine scharf berandete, lateral von einem zarten Sklerosesaum umgebene Osteolyse an der medialen distalen Femurmetaphyse (Lodwick-Grad IC). In der MRT wird eine unscharf abgrenzbare, die Kortikalis überschreitende Tumorformation mit Bluteinlagerungen und einem deutlichen Umgebungsödem sichtbar (Abb. 2). Bei genauer Betrachtung der konventionellen Röntgenaufnahme sind zusätzlich aber auch noch strukturelle Knochenveränderungen sichtbar: Die Epiphysenfugen um das Kniegelenk sind aufgeweitet, die sich anschließende Metaphysenregion erscheint unscharf und die Spongiosa ist insgesamt netzigwabig transformiert.

Aufgrund dieser Röntgenzeichen stellten wir die Diagnose eines braunen Tumors bei Rachitis mit regulativem Hyperparathyreoidismus. Zusätzlich angefertigte

Fortschr Röntgenstr 2000; 172: 714-715 (c) Georg Thieme Verlag Stuttgart · New York ISSN 1438-9029

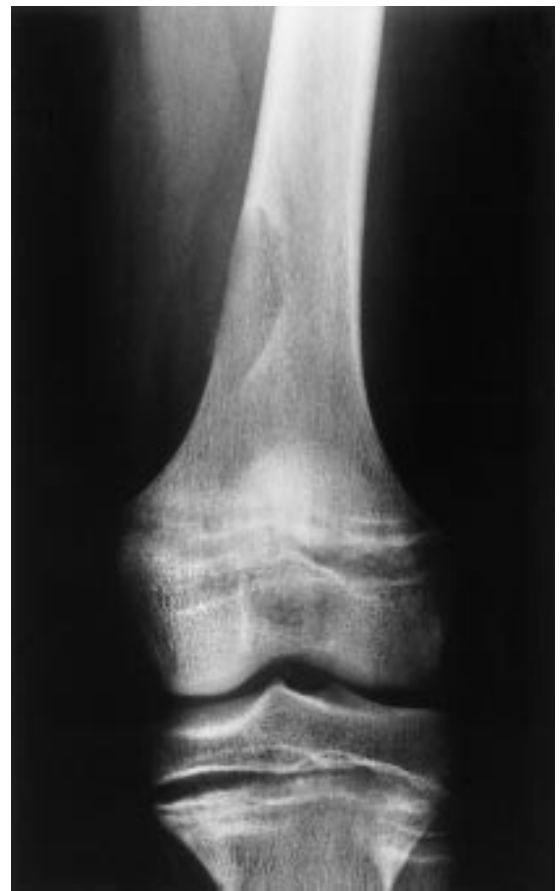

Abb. 1 Linkes Knie a.p. bei Vorstellung der Patientin: Scharf berandete Osteolyse an der medialen Femurmetaphyse mit lateralseitigem zarten Sklerosesaum, zusätzlich erweiterte Epiphysenfugen.

Handaufnahmen bestätigten unsere Diagnose (Abb. 3).

Die ergänzende klinische Untersuchung fand in der Kinderklinik statt, die dort bestimmten Laborparameter zeigten eine deutliche Erhöhung der alkalischen Phosphatase sowie eine mäßige Erhöhung des Parathormons und erniedrigtes anorganisches Phosphat und 25-OHVitamin D. Ein differentialdiagnostisch in Frage kommender primärer Hyperparathyreoidismus und ein Phosphatdiabetes konnten ausgeschlossen werden. Als Therapie wurde eine hochdosierte Vitamin D-Substitution eingeleitet.

In der Verlaufskontrolle nach dreimonatiger Therapie (hier nicht abgebildet) haben sich die klaffenden Epiphysenfugen und die netzig-wabige Spongiosatransformation leicht zurückgebildet, um den durchgebrochenen braunen Tu-
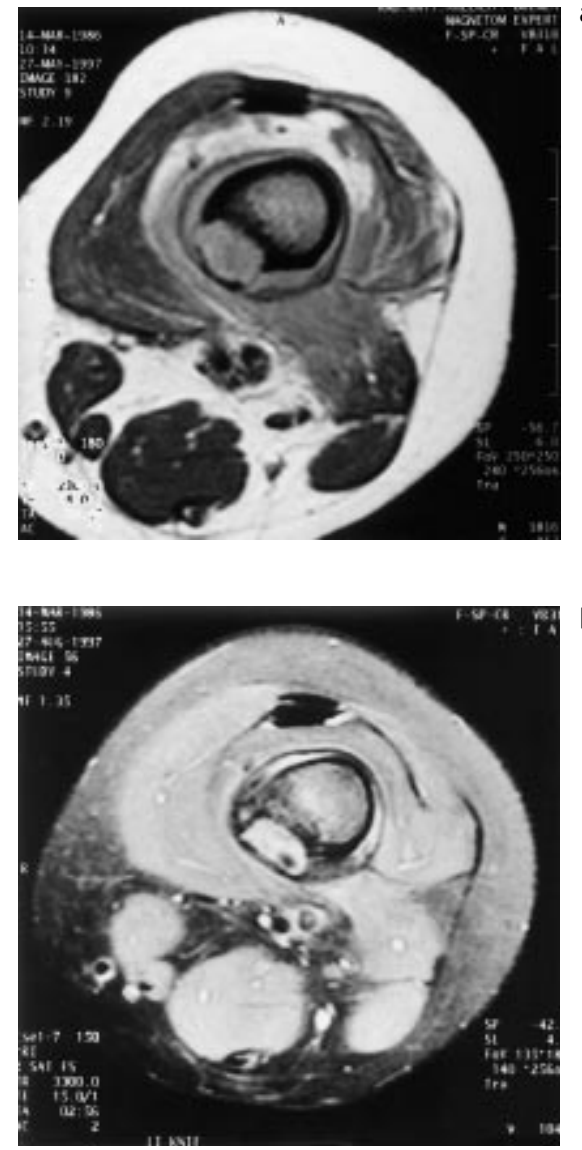

Abb. 2 Das axiale protonengewichtete (a) und fettgesättigte $T_{2}$ (b) MR-Bild zeigt einen die Kortikalis überschreitenden Tumor mit Einblutung sowie ein deutliches Ödem der umgebenden Muskulatur wie bei einem aggressiven Tumor.

mor hat sich eine solide Periostmanschette formiert. Insgesamt also immer noch ein eindeutig pathologischer Befund, so dass die Vitamin D-Gabe fortgesetzt wurde.

In einer nach 18 Monaten durchgeführten Kontrolle ist die ehemalige Tumorregion nun komplett knöchern durchbaut und die Epiphysenfugen sind wieder altersentsprechend konfiguriert ohne Zeichen einer Rachitis oder eines Hyperparathyreoidismus (Abb.4). Die Patientin ist klinisch komplett beschwerdefrei.

\section{Diskussion}

Ein brauner Tumor ist eine bei uns heute nur noch selten anzutreffende Manifestationsform des Hyperparathyreoidismus. Im hier vorgestellten Fall ist er auf dem Boden einer sogenannten Immigranten-Rachitis mit reaktivem Hyperparathyreoidismus entstanden. Wenn 


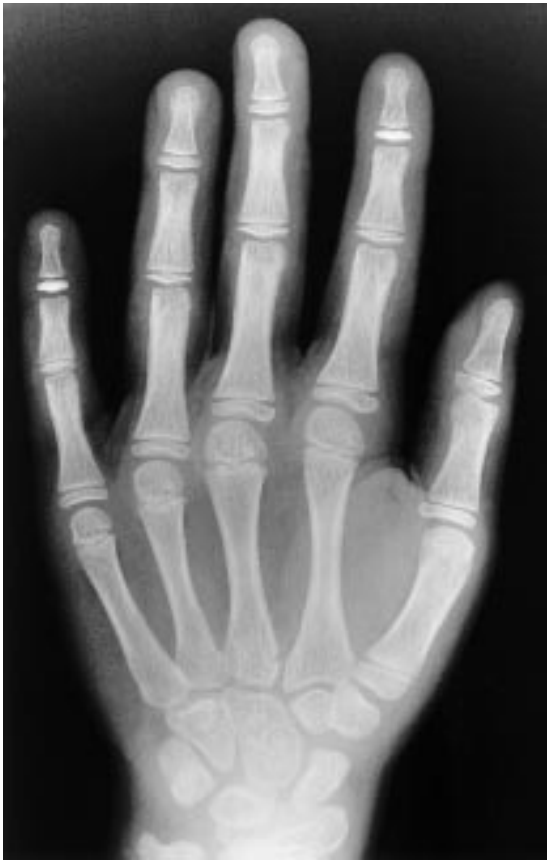

Abb. 3 Klassisches Bild einer Rachitis mit regulativem Hyperparathyreoidismus mit „gebecherten“ metakarpalen Epiphysenfugen. Die Kompakta der Akren ist dünn und aufgebessert, es besteht eine netzig-wabige Spongiosararifizierung.

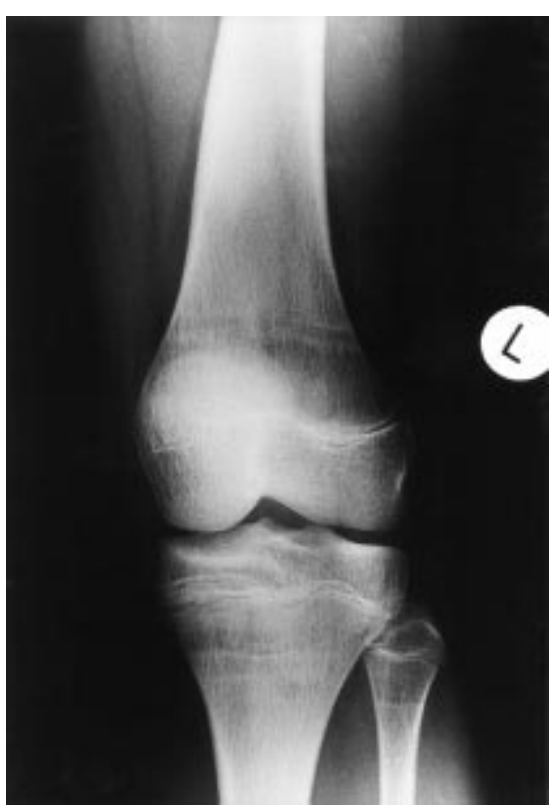

Abb. 4 In der Kontrolle 18 Monate nach Diagnosestellung und nach fortgesetzter Vitamin D-Substitution komplette knöcherne Durchbauung der ehemaligen Tumorregion und normale Konfiguration der Epiphysenfugen.
Kinder aus dem arabischen Kulturkreis auch in Nordeuropa ihre übliche Bekleidungsweise mit Einhüllen in lange Kleider und Tragen von Kopftüchern beibehalten, wird fast jegliche Sonnenbestrahlung der Haut verhindert und so die durch UV-Strahlung ausgelöste Umwandlung von 7-Dehydrocholesterol in stoffwechselaktives Cholecalciferol in der Haut unmöglich. Hinzu kommt noch eine verminderte orale Aufnahme von Vitamin D durch andere Ernährungsgewohnheiten. Durch den so entstandenen langanhaltenden Vitamin D-Mangel kann sich ein regulativer Hyperparathyreoidismus entwickeln, in dessen Folge schließlich auch braune Tumoren auftreten können.

Der braune Tumor hat sich in unserem Fall bemerkenswerterweise an der distalen medialen Femurmetaphyse gebildet, genau dort, wo der Knochen durch den Ansatz des M.adductor magnus einer erhöhten mechanischen Belastung ausgesetzt ist.

Die Metaphysen der Kniegelenksregion sind andererseits beim gesunden Knochen der typische Ort für die Entwicklung von fibrösen metaphysären Defekten (FMD) - eine der häufigsten tumorähnlichen Läsionen des kindlichen Skeletts, die bei etwa drei bis vier Prozent aller Heranwachsenden vorkommen (Freyschmidt J. et al., Knochentumoren, Springer 1998, 680). Sie entsprechen einer fokalen Wachstumsstörung, die offensichtlich Folge vermehrter Umbauvorgänge des Knochens durch starke Traktion im Bereiche von Muskelinsertionen ist (Ritschl P. et al., Skeletal Radiol 1989; 18: 253). Nur selten kommt es bei größeren Fibromen zu Spontanfrakturen oder zu Einbrüchen, die dann zu einer veränderten Röntgensymptomatik führen, die wiederum einen aggressiven Prozess vortäuschen kann (Tigges S. et al., Amer J Roentgenol 1995, 165: 143).

Die lokalisatorische Deckungsgleichheit zwischen einem FMD im distalen Femur und dem in unserem Fall aufgetretenen braunen Tumor lässt den Schluss zu, dass zu seiner Entstehung ein mechanischer Reiz als Kofaktor vorgelegen hat, der zu einer überstarken lokalen Stimulation der osteoklastären Resorption führte und damit zur Pseudotumorbildung genau an diesem Skelettabschnitt. Die Tatsache der vollständigen knöchernen Durchbauung nach Vitamin D-Substitution in einem Zeitraum von 18 Monaten ohne irgendwelche Residuen beweist letztendlich auch, dass der von uns unterstellte braune Tumor nicht in einem vorexistenten nichtossifizierenden Knochenfibrom entstanden ist.

Ein weiterer Grund dieser Fallvorstellung war der „alarmierende“ MR-tomographische Befund mit deutlicher ödematöser Reaktion und Einblutung in das umgebende Weichgewebe, der primär zur Annahme eines malignen Knochentumors geführt hatte. Dieser tumorsimulierende Charakter erklärt sich zwanglos aus der Pathogenese brauner Tumoren: Spongiosa und Kortikalis werden durch den von der Parathormonausschüttung stimulierten osteoklastären Knochenabbau lokal zerstört, es entstehen Höhlen, in die es einbluten kann. Eine komplette Kortikaliszerstörung führt, wie bei unserer Patientin, zu einer Blutung aus dem Knochen in die Umgebung und es entsteht bei alleiniger Betrachtung dieses Befundes ein in die Irre führendes radiologisches Leitsymptom.

Im vorliegenden Falle weisen die weiten und „gebecherten“ Epiphysenfugen um das Kniegelenk und die Spongiosatransformation eindeutig auf eine Rachitis mit sekundärem Hyperparathyreoidismus hin und es liegt nahe, diese Röntgenzeichen mit der „tumorösen“ Läsion zu verknüpfen. Eine einfache Röntgenaufnahme der Hand mit Darstellung spezifischer Veränderungen (Zeichen der Rachitis und des Hyperparathyreoidismus, Abb. 3) gibt dem Radiologen schnell zusätzliche Sicherheit und die radiologische Diagnose bedarf dann nur noch einer laborchemischen Bestätigung.

Durch die synoptische Betrachtungsweise kann eine überflüssige Biopsie verhindert werden.

A. Sternberg, J. Freyschmidt, Bremen 